\title{
Fusobacterium necrophorum infections in England and Wales 1990-2000
}

\author{
J. S. BRAZIER, V. HALL, E. YUSUF* and B. I. DUERDEN
}

PHLS Anaerobe Reference Unit, Department of Medical Microbiology and Public Health Laboratory, University of Wales College of Medicine, Heath Park, Cardiff CF14 4XW and *PHLS Communicable Disease Surveillance Centre, 61 Colindale Avenue, London NW9 5EQ

\begin{abstract}
In response to a marked increase in both the number of Fusobacterium necrophorum bacteraemia reports to the PHLS Communicable Disease Surveillance Centre and the number of $F$. necrophorum isolates referred to the PHLS Anaerobe Reference Unit in 1999, the data from both sources on $F$. necrophorum infections were reviewed for the decade 1990-2000. There were 208 reports of $F$. necrophorum bacteraemia (average 19/year; range 14-34/year) with a peak in incidence in the late winter months; $68 \%$ were from male patients and the peak age range was 16-23 years. Of 205 referred isolates of $F$. necrophorum, $122(59 \%)$ were from blood cultures and these represented $58 \%$ of the bacteraemia reports; the others were from brain and soft tissue abscesses, pleural and joint fluids, eyes, ears and lymphatic tissue. The average number of referrals was 19/year (range 9-37/year). The peak year for bacteraemia reports (34) and isolate referrals (37) was 1999; this increase was not sustained in 2000. All isolates were susceptible to metronidazole, but $2 \%$ were resistant to penicillin and $15 \%$ to erythromycin. $F$. necrophorum continues to be a regular but uncommon cause of bacteraemia and metastatic abscesses following an acute sore throat, especially in young, otherwise healthy adults.
\end{abstract}

\section{Introduction}

Human infection with Fusobacterium necrophorum, an obligately anaerobic gram-negative bacillus, has been known since 1898 [1]. These are often serious infections that can be potentially life-threatening. Unlike most non-sporing anaerobic pathogens, F. necrophorum is not solely an opportunist pathogen that causes disease in patients made susceptible by underlying disease or surgery. Although it forms part of the normal flora of the oral cavity, it can become a primary pathogen that causes severe disease in otherwise healthy individuals. F. necrophorum causes disease in man and a wide range of animals, often described collectively as necrobacillosis, but the human and animal strains are distinct [2-4], as are the diseases caused.

The clinical characteristics of classical F. necrophorum infection in man were first described by the French clinician Lemierre, in 1936 [5]. He described a series of 18 deaths due to anaerobic septicaemia caused by $F$.

Received 14 Aug. 2001; accepted 24 Sept. 2001.

Corresponding author: Professor B. I. Duerden (e-mail: bduerden@phls.org.uk). necrophorum with a syndrome 'so characteristic that mistake is almost impossible ... It becomes relatively easy to make a diagnosis on clinical findings before bacteriological examination, including blood culture, has provided conclusive proof'. This condition usually affects previously healthy young adults and there are no known predisposing factors. Patients present with an acute sore throat with purulent exudate, a pseudomembrane over the tonsils, cervical and submandibular lymphadenopathy and a high fever with rigors, which may progress to septic shock. Metastatic abscesses in the liver, kidneys and lungs, pyogenic arthritis and osteomyelitis are all common [5-7]. However, even today, a lack of awareness of the classical symptoms of Lemierre's syndrome (necrobacillosis) amongst clinicians in different specialities leads to delayed or missed diagnosis and inappropriate treatment. Indeed, the condition seems so remarkable to succeeding generations of physicians that descriptions and reports of small collections of cases of this 'unusual and dramatic' infection have appeared regularly over the last half century [6-11]. Without antibiotic treatment, the mortality is high, but with appropriate antibiotics (e.g., penicillin, metronidazole), the response is generally good with full recovery. 


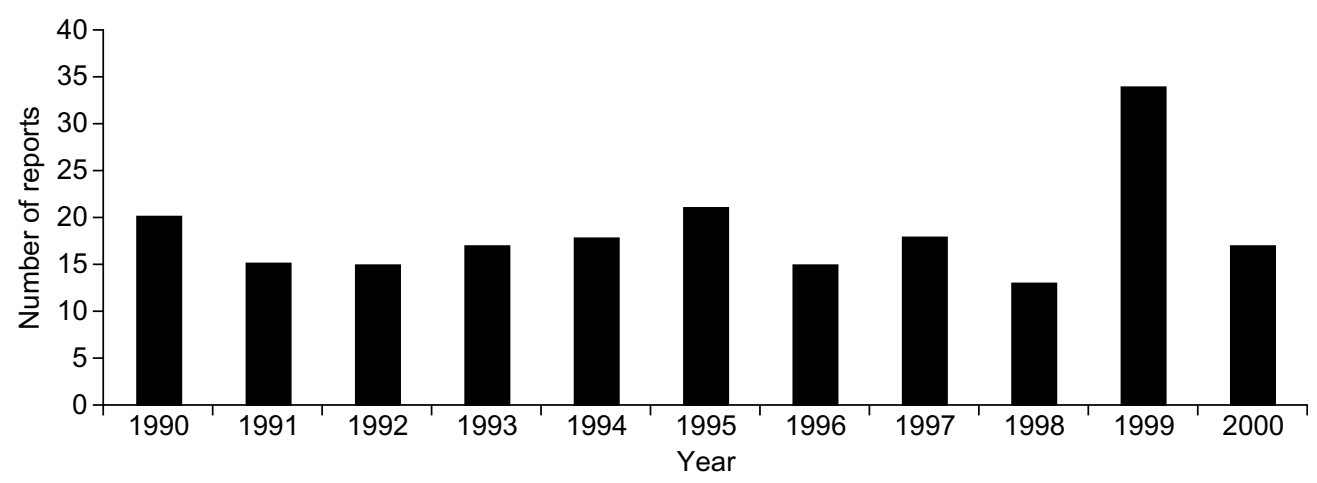

Fig. 1. F. necrophorum bacteraemias in England and Wales reported to CDSC 1990-2000 ( $\mathrm{n}=208)$.

Difficulties in isolation and identification of $F$. necrophorum may also confound laboratory diagnosis. However, alert diagnostic microbiology laboratories with good anaerobic methods, including anaerobic blood cultures, are able to isolate this organism and laboratories in England and Wales commonly refer isolates to the PHLS Anaerobe Reference Unit (ARU) at Cardiff Public Health Laboratory for identification. The laboratories are also asked to report all incidents of bacteraemia to the PHLS Communicable Disease Surveillance Centre (CDSC), Colindale, London, and this includes reporting of $F$. necrophorum bacteraemia.

In 1999, there was an increase in the number of $F$. necrophorum isolates referred to ARU and a parallel increase in reports of $F$. necrophorum bacteraemia to CDSC. This report combines these data for the decade 1990-2000 to determine whether there has been a true increasing trend in this infection.

\section{Materials and methods}

\section{Bacteraemia reports}

All reports of $F$. necrophorum bacteraemia received by CDSC from laboratories in England and Wales during the period 1990-2000, inclusive, were analysed by year, time of year (quarters) and by age and sex of the patients.

\section{Referrals to $A R U$}

All records of isolates referred to the ARU that were identified as $F$. necrophorum were analysed by year, site of infection (blood or other clinical sites) and antimicrobial susceptibility determined by disk sensitivity tests [12] to metronidazole, penicillin, tetracycline, erythromycin, clindamycin and chloramphenicol. The 77 isolates received from 1997 onwards were also tested against amoxicillin/clavulanate, imipenem and cefoxitin. Patient demographic data were not uniformly available and, therefore, were not analysed.

\section{Results}

\section{F. necrophorum bacteraemia}

A total of 208 bacteraemias due to F. necrophorum was recorded by CDSC during the period 1990-2000. The annual number of reported $F$. necrophorum bacteraemias ranged from a low of 14 in 1998 to a peak of 34 in 1999. The annual average number of cases was 19

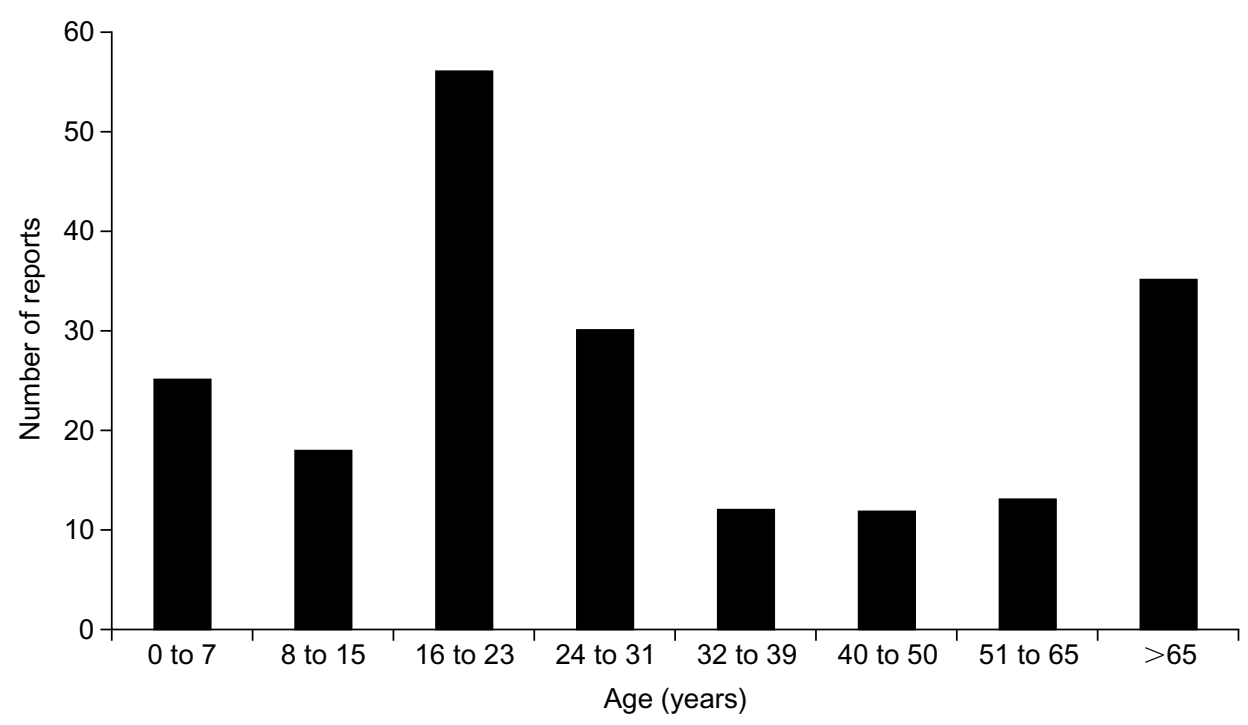

Fig. 2. F. necrophorum bacteraemias in England and Wales reported to CDSC by age during 1990-2000 $(\mathrm{n}=208)$. 


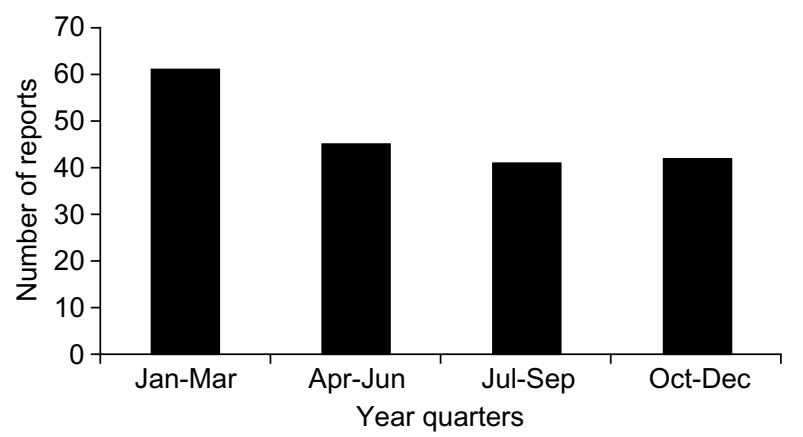

Fig. 3. Seasonal distribution of F. necrophorum bacteraemias 1990-2000.

(Fig. 1). Analysis of patient data revealed a marked significant difference in the sex distribution of cases; $143(68 \%)$ were males and $64(31 \%)$ females with 1 unknown $(p<0.0001)$. The age range of cases peaked among adolescents and young adults in the 16-23 year old age group. This group accounted for 57 (30\%) of 188 cases in which the age of the patient was known. The next highest age band was the over $65 \mathrm{~s}$ with 35 $(19 \%)$ cases (Fig. 2). There was an overall peak in incidence in the first quarter of the year (Fig. 3).

\section{Referrals of F. necrophorum to ARU}

Over the same period there were 205 referrals to the ARU of isolates from human clinical sources that were identified as F. necrophorum. Of these, 122 (59\%) were from blood cultures and these are most probably all duplicated in the reports of $F$. necrophorum bacteraemias to CDSC. Therefore, it can be estimated that the ARU received 58\% (122/205) of all isolates of $F$. necrophorum from blood cultures for confirmation. The remaining 83 referred isolates were from a range of clinical sites of infection including: brain and other soft tissue abscesses, pleural fluids, joint fluids, eyes, ears, wounds and lymphatic tissue. The number of referrals per year over the decade 1990-2000 ranged from nine in 1991 to a peak of 37 in 1999 with an annual average of 19 (Fig. 4). Data on the susceptibility of 100 of the 205 isolates of $F$. necrophorum to a range of antimicrobial agents are on record and have been analysed. No resistance was recorded to metronidazole, a key drug for treatment of fusobacterial infections, nor to amoxicillin/clavulanate, cefoxitin, chloramphenicol, clindamycin or imipenem. Fifteen (15\%) isolates showed either resistance or reduced sensitivity to erythromycin, $2(2 \%)$ resistance to penicillin and $1(1 \%)$ resistance to tetracycline.

\section{Discussion}

Several workers noted that Lemierre's disease was sparsely reported in the 1960s and 1970s. They postulated that the widespread use of penicillin, prescribed mainly as an antistreptococcal agent for sore throats, may have accounted for this. Equally, the poor standard of anaerobic bacteriology generally practised at that time may have played a part in the under-recognition of fusobacterial disease in the laboratory. During the 1980 s and 1990s, reports of Lemierre's disease often referred to it as 'the forgotten disease' [7-9] as it became more commonly recognised. Again, improved awareness and better anaerobic methods probably played a part.

It has been postulated that the restricted use of antibiotics prescribed for the treatment of sore throats and tonsillitis that present to general practitioners (GPs) may account for an increase in the number of cases of Lemierre's disease $[10,11]$. Certainly, general practitioners in England and Wales, under guidance from such reports as 'The Path of Least Resistance' [13] and other material issued by the Department of Health in England, have recently been advised to reduce their prescribing of antibiotics for conditions believed to be primarily of viral aetiology, such as sore throats. Furthermore, the incidence of $15 \%$ resistance to erythromycin, an agent widely recommended for respiratory tract infections in primary

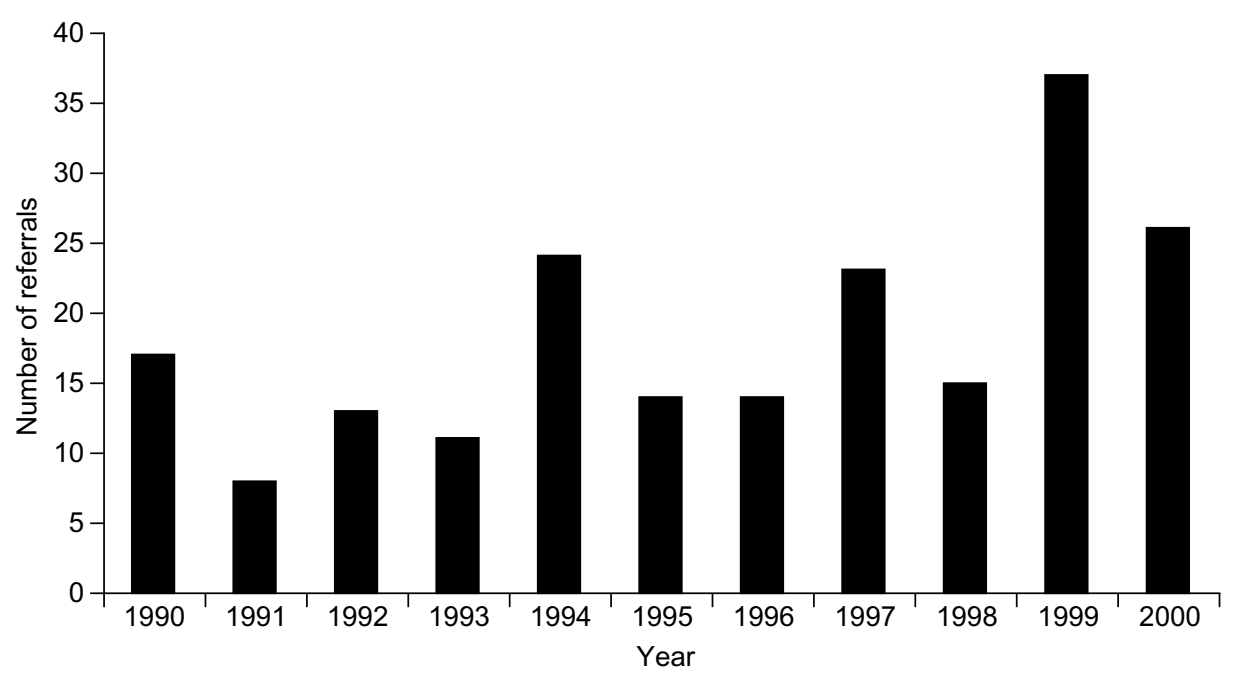

Fig. 4. Referrals of F. necrophorum to the Anaerobe Reference Unit from all sources 1990-2000 (n = 205). 
care, is of potential significance. It would certainly be of interest to determine the level of erythromycin prescribing as a potential risk factor for the development of sepsis with $F$. necrophorum in young adults.

If the data presented here had stopped in 1999, there would have been continued support for the theory that prescribing practices may play a role, because both CDSC and ARU saw unusually high numbers of $F$. necrophorum bacteraemias and F. necrophorum referrals, respectively, during 1999. However, this upward trend was not maintained in 2000, when reports of bacteraemias returned to the 10-year average and referrals to ARU also fell but not to the average of previous years. Nevertheless, clinicians and microbiologists should remain vigilant. In the light of previous knowledge and the data presented here, GPs should be aware that some cases of acute sore throats or tonsillitis in teenagers and young adults, especially males, in the late winter months, may progress to serious disease if untreated.

\section{References}

1. Hallé J. Recherches sur la bacteriologie du canal génital de la femme (état normal et pathologique). Thèse de Paris, Steinheil. 1898.
2. Hall V, Duerden BI, Magee JT, Ryley HC, Brazier JS. A comparative study of Fusobacterium necrophorum strains from human and animal sources by phenotypic reactions, pyrolysis mass spectrometry and SDS-PAGE. J Med Microbiol 1997; 46: $865-871$.

3. Brown R, Lough HG, Poxton IR. Phenotypic characteristics and lipopolysaccharides of human and animal isolates of Fusobacterium necrophorum. J Med Microbiol 1997; 46: 873-878.

4. Smith GR, Thornton EA. Classification of human and animal strains of Fusobacterium necrophorum by their pathogenic effects on mice. J Med Microbiol 1997; 46: 879-882.

5. Lemierre A. On certain septicaemias due to anaerobic organisms. Lancet 1936; 1: 701-703.

6. Alston JM. Necrobacillosis in Great Britain. BMJ 1955; 2: $1524-1528$.

7. Moore-Gillon J, Lee TH, Eykyn SJ, Phillips I. Necrobacillosis: a forgotten disease. BMJ 1984; 288: 1526-1527.

8. Weesner CL, Cisek JE. Lemierre syndrome: the forgotten disease. Ann Emerg Med 1993; 22: 256-258.

9. Koay CB, Heyworth T, Burden P. Lemierre syndrome: a forgotten complication of acute tonsillitis. $J$ Laryngol Otol 1995; 109: 657-661.

10. Leugers CM, Clover R. Lemierre's syndrome: postanginal sepsis. J Am Board Fam Pract 1995; 8: 384-391.

11. Lustig LR, Cusick BC, Cheung SW, Lee KC. Lemierre's syndrome: two cases of postanginal sepsis. Otolaryngol Head Neck Surg 1995; 112: 767-772.

12. Duerden BI. Role of the reference laboratory in susceptibility testing of anaerobes and a survey of isolates referred from laboratories in England and Wales during 1993-1994. Clin Infect Dis 1995; 20: S180-S186.

13. The Path of Least Resistance: Report of the Standing Medical Advisory Committee Sub-Group on Antimicrobial Resistance. London, Department of Health. 1998. 